\title{
Comparison of Simple Communication Aids between Amyotrophic Lateral Sclerosis Patients and Caregivers
}

\author{
Tomoya Miyasaka, Mamiko Kamoshida, Norio Kato, Keiko Kawashima
}

\begin{abstract}
Amyotrophic lateral sclerosis (ALS) is a neuromuscular disease in which muscle strength and motor function decrease with the progress of the disease. As the decreased motor function is associated with poorer communication abilities in ALS patients, supporting methods are needed to enable better communication according to their residual capacity. The purpose of this study was to evaluate and compare features of the "transparent character board" and the "mouth-shape character", which are simple communication methods for patients with ALS and other diseases in which patients have difficulty in speaking or writing. Healthy subjects role-played the patient (the patient's role) and the caregiver (the caregiver's role). The trials were conducted to express the intention of the patient using Hiragana characters (The Japanese alphabet) to the caregiver using 3 communication aids: a "transparent character board", the "mouth-shape characters" method, and an "electronic character boards" with voice output set as the control. Each trial was recorded with an eye-tracking device worn by the caregiver and a video camera, and the communication process was measured. The transparent character board communication requires the board as a tool. This method can be used for speedy communication if the environment allows the patient and caregiver to spend much time learning the operation process. The mouth-shape character method requires no tools for communication. The ease of the method makes it applicable even when the relationship between the patient and caregiver is not so long.
\end{abstract}

Index Terms - Amyotrophic lateral sclerosis, communication support, Transparent character board, Mouth-shape character

\section{INTRODUCTION}

Amyotrophic lateral sclerosis (ALS) is a neuromuscular disease [1] in which muscle strength [2] and motor function decrease with the progress of the disease. As the decreased motor function is associated with poorer communication abilities in ALS patients [3], supporting methods are needed to enable better communication according to their residual capacity [4]. Although advanced techniques have been proposed to support communication [5]-[7], few of them are widely used in clinical and home care settings, and communication is often conducted in a very simple manner.

Tomoya Miyasaka, Department of Physical Therapy, Faculty of Health Sciences, Hokkaido University of Science, Sapporo, Hokkaido, Japan.

Mamiko Kamoshida, Department of Physical Therapy, Faculty of Health Sciences, Hokkaido University of Science, Sapporo, Hokkaido, Japan.

Norio Kato, Department of Physical Therapy, Faculty of Health Sciences, Hokkaido University of Science, Sapporo, Hokkaido, Japan.

Keiko Kawashima, Department of Physical Therapy, Faculty of Health Sciences, Hokkaido University of Science, Sapporo, Hokkaido, Japan.
These aids include transparent character boards [8] and simpler, the tool-less mouth-shape character method, developed in recent years [9]. A number of studies have been reported related to communication aids with ALS patients, but few have made quantitative evaluations.

The purpose of this study was to evaluate and compare features of the "transparent character board" and the "mouth-shape character", which are simple communication methods for patients with ALS and other diseases in which patients have difficulty in speaking or writing. In addition, the results were examined, and the effective use of these methods in clinic and home care settings is presented.

\section{METHOD}

The experimental methods were referred to in the previous studies [10]. Healthy subjects role-played the patient (the patient's role) and the caregiver (the caregiver's role). The trials were conducted to express the intention of the patient using Hiragana characters (The Japanese alphabet) [11] to the caregiver using 3 communication aids: a "transparent character board", the "mouth-shape characters" method, and an "electronic character boards" with voice output set [12] as the control. Each trial was recorded with an eye-tracking device [13] worn by the caregiver and a video camera, and the communication process was measured. The patient was assumed to have loss of upper limb motor function, loss of articulatory and voice functions, functional voluntary contraction around the face such as eyelids and facial muscles, normal visual and auditory sensory functions, and normal cognitive levels.

\section{A. Chart of Hiragana characters}

Hiragana characters, one of the Japanese phonetic alphabets,

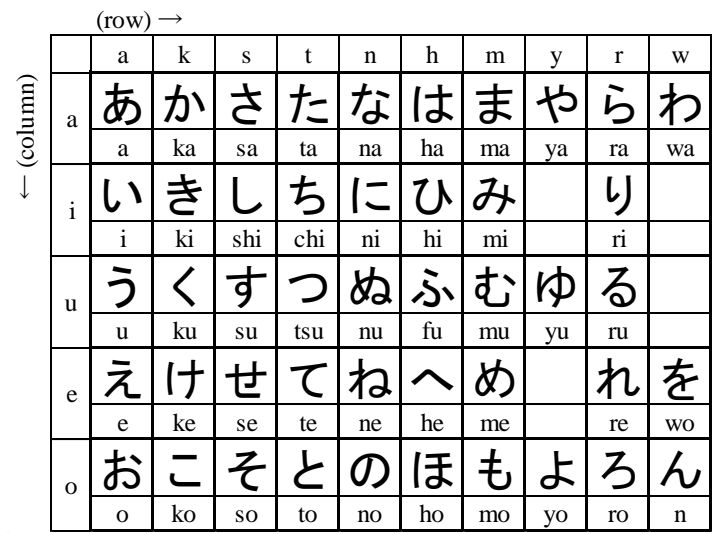

Fig. 1 Chart of Hiragana characters 
were used in this experiment. Hiragana characters were represented in chart form consisting of 50 character boxes in which consonants of the same series are arranged in 10 columns and vowels are arranged in 5 rows (Fig. 1).

\section{B. "Transparent character board"}

A transparent character board was prepared by printing the Chart of Hiragana characters on a transparent resin plate (Fig. 2). The patient and the caregiver were paired, and the caregiver communicated with the patient by confirming the intention of the patient transmitted through the Hiragana characters. The caregiver should not speak. Using the transparent character board to identify the Hiragana intended by the patient required the following 8 processes.

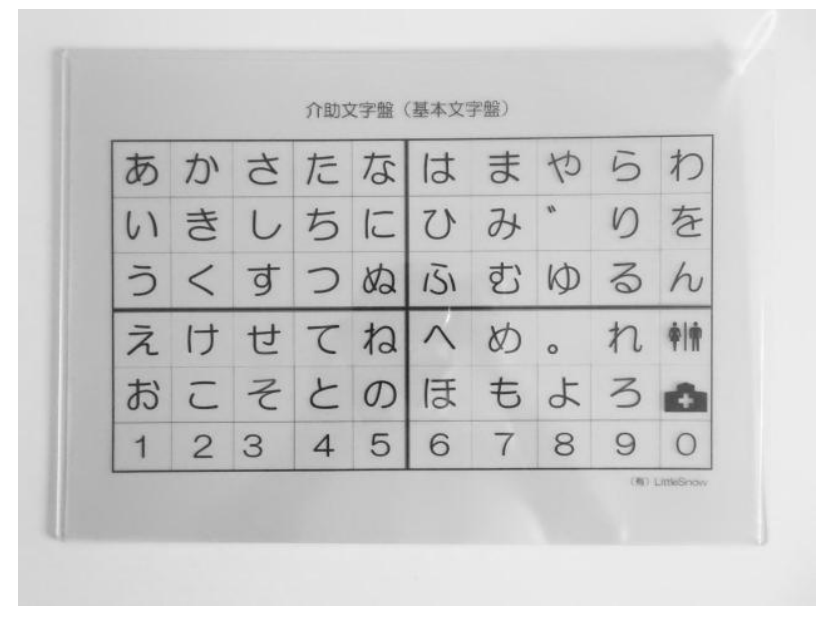

Fig. 2 Transparent character board

1) The patient and the caregiver faced each other. The caregiver held the transparent character board with both arms $0.4 \mathrm{~m}$ away from his/her body, and the board was placed between the two persons. To enable the patient to see the Hiragana in the correct direction, the transparent character board was oriented such that the character "a" at the top left on the chart was above the caretaker's right hand.

2) Through the "a" box on the transparent character board, the lines of sight of the caregiver and the patient were matched.

3) The caregiver moved the transparent character board to the right from "a" to the "ka", "sa" and so on, so that the lines of sight of both persons could pass the top character box in the each column.

4) When the line of sight of the caregiver entered the top character box in the column that included the Hiragana intended by the patient, the patient signaled to the caregiver by moving his/her facial muscles.

5) The caregiver confirmed the intention of the patient and identified the column.

6) The caregiver moved the transparent character board upward so that the line of sight of the patient entered the identified column.

7) When the line of sight of the caregiver entered the character box intended by the patient, the patient signaled to the caregiver by moving his/her facial muscles.

8) The caregiver confirmed the intention of the patient and identified the Hiragana.

\section{C. "Mouth-shape character"}

There are two ways of communication using the mouth-shape character method (Fig. 3). One is to start by shaping the mouth for vowel production from a patient, and the other is to start by vocalization from a caregiver. This study employed the latter method. The patient and the caregiver were paired, and the caregiver confirmed the intention of the patient expressed by the Hiragana character, and communicated the intention. Using the mouth-shape character method to identify the Hiragana intended by the patient required the following 7 processes.

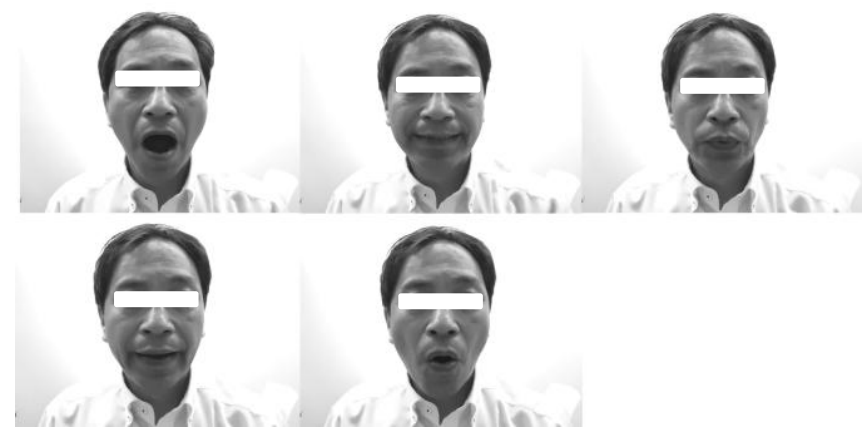

Fig. 3 Mouth-shape character

1) The patient and the caregiver faced each other so that each of his/her facial expressions could be confirmed by the other.

2) The caregiver vocalized clearly the Hiragana of the top character in the each column from left to right, "a", "ka", "sa" and so on and by moving his/her mouth to make it easier for the patient to understand.

3) When the caregiver vocalized the top character in the column that included the Hiragana intended by patient, the patient signaled to the caregiver by moving his/her facial muscles.

4) The caregiver confirmed the intention of the patient and identified the column.

5) The caregiver vocalized the characters in the identified column from the top to the bottom according to the chart of Hiragana characters.

6) When the caregiver vocalized the character intended by the patient, the patient signaled to the caregiver by moving his/her facial muscles.

7) The caregiver confirmed the intention of the patient and identified the Hiragana.

\section{Electric character board}

An electronic character board (Let's Chat, Panasonic Inc., Japan) with voice output was used as a control (Fig. 4). Using the electronic character board, the caregiver pressed the push button switches of the external input, and scanned the columns/characters and confirmed the Hiragana. The scanning speed of the one column and the one character was 1 process/s. Using the electric character board to identify the Hiragana intended by the patient required the following 8 processes.

1) The electronic character board was placed in front of the caregiver with the board visible to the patient.

2) The caregiver pressed the push button switch. Then, the 
board was scanned one column at a time while vocalizing 5 Hiragana characters composing the individual column, such as "a-i-u-e-o", "ka-ki-ku-ke-ko". "sa-shi-su-se-so" and so on.

3) When the board vocalized the character column that included the Hiragana intended by patient, the patient signaled to the caregiver by moving his/her facial muscles.

4) The caregiver confirmed the intention of the patient and identified the column by pressing the push button switch.

5) The board scanned five characters within the identified column while vocalizing every single character at a time.

6) When the board vocalized the character that included the character intended by patient, the patient signaled to the caregiver by moving his/her facial muscles.

7) The caregiver confirmed the intention of the patient and identified the Hiragana by pressing the push button.

8) The board vocalized the identified character and recorded it on the device.

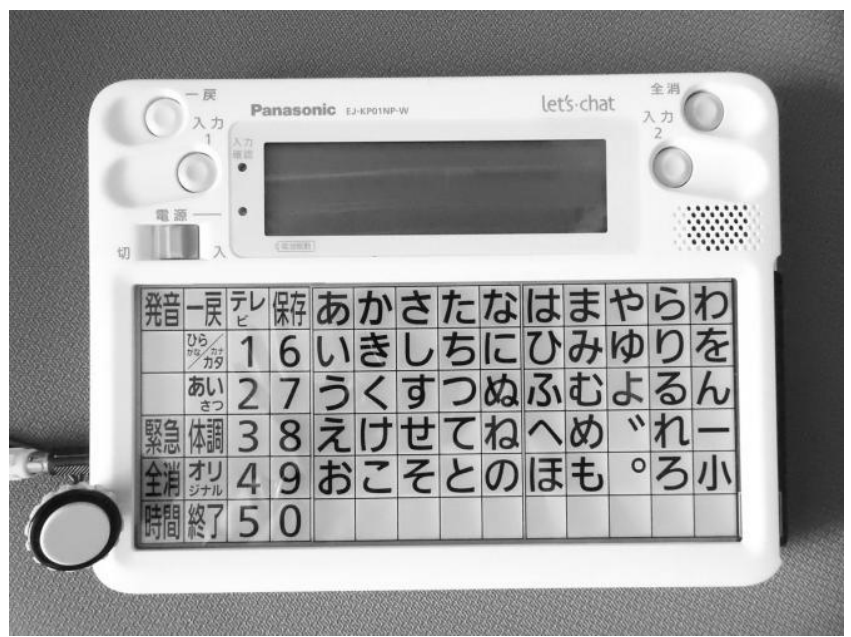

Fig. 4 Electric character board

\section{E. Experimental}

Ten pairs of Hiragana characters expressed by the intention of patient, consisting of 3 characters that are not found in Japanese words, were created. The order of the 10 pairs were randomized. Subjects were 4 healthy persons over 20 years old. They were given the roles of patient and caregiver, facing each other in pairs and sitting in chairs at a distance of $1.2 \mathrm{~m}$. For each of the 3 communication aids, one subject performed a total of 30 Hiragana character communication trials consisting of 10 pairs times 3 characters (Fig. 5-7). A total of 120 characters were trialed for each communication aid. None of the subjects had any previous experience with the trials, and this study was conducted with prior explanation from the researchers. When the communication trial failed, the number of failures was counted and the trials were carried out again using the same Hiragana characters.

\section{F. Evaluation methods}

The communication process was recorded with a video camera placed on the side of the subjects facing each other and an eye-movement measuring device (T.K.K. 2951, Takei Scientific Instruments Co., Ltd., Japan) (Fig. 5 -7) was attached to the caregiver. The number of successes and failures in communication was recorded, and the subjects were asked for their impressions after the trials.

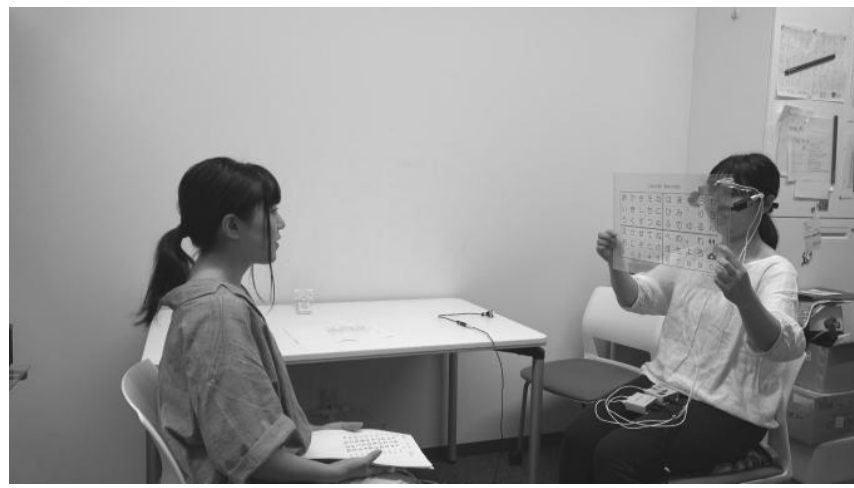

Fig.5 Communication Experiments; Transparent character board

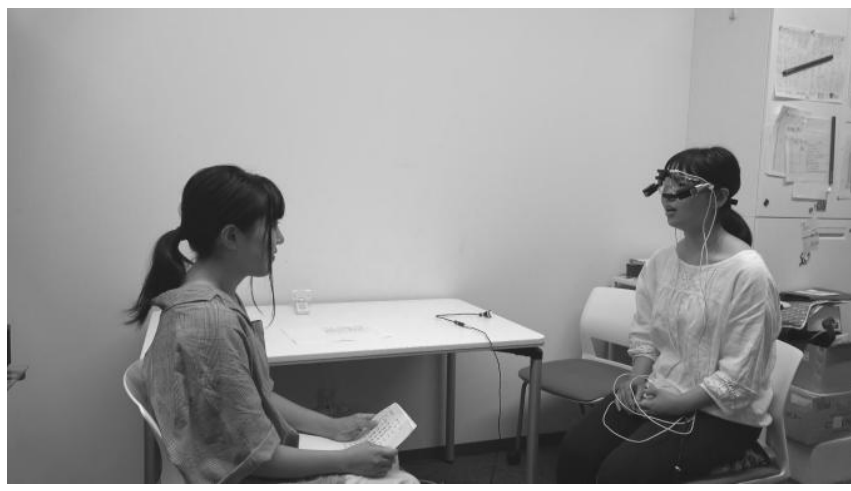

Fig.6 Communication Experiments; Mouth-shape character

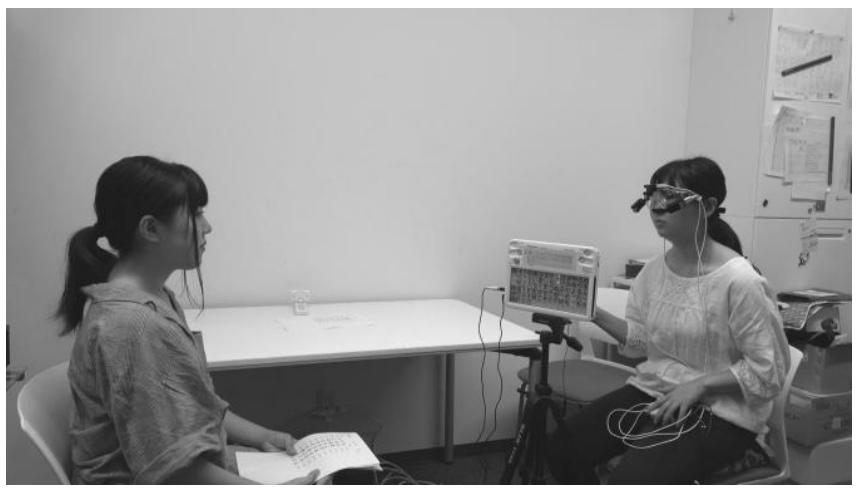

Fig.7 Communication Experiments; Electronic character board

Because the processes to reach the target column and the row were different for every Hiragana character on the board, the process number was set for each one. For example, "su" was positioned in a "sa" column (the 3rd column from left) and a "u" row (3rd row from top), and the process number was set to 6 as the sum of the number of columns and rows. Similarly, since "me" was positioned in a "ma" column (the 7th column from left) and an "e" row (4the row from top), the process number was set to 11 . A video file recorded from the video camera and the eye-movement measuring device was 
reproduced, and the process number of characters and the process time were totaled.

\section{G. Research Ethics}

This study was conducted based on the Research Ethics Application No. 407 of Hokkaido University of Science after subjects had been informed in advance of the research and given their consent. The experiment was carried out ensuring the safety of the subjects.

\section{RESUltS}

The process number and the time required for the 3 communication aids are shown in Fig. 8-10. The process number was the same for all 3 types of trials, and the process number was $8 \pm 2.78(\mathrm{~N})$ with the minimum value of $2(\mathrm{~N})$ and the maximum value of $12(\mathrm{~N})$. The time of the transparent character board was $8.51 \pm 4.41$ (s) with the minimum value of 1.4 (s) and the maximum value of 20.17 (s). The time of the mouth-shape character method was $8.16 \pm 3.26$ (s) with the minimum value of 1.6 (s) and the maximum value of 15.9 (s). The time of the electronic character board was $7.89 \pm 2.83$ (s) with the minimum value of 2.1 (s) and the maximum value of 15.17 (s). The time was totaled on the trials which succeeded in the communication. The number of communication failures was 5 times for the mouse-shape character method, 13 times for the transparent character board, and 6 times for the electronic character board.

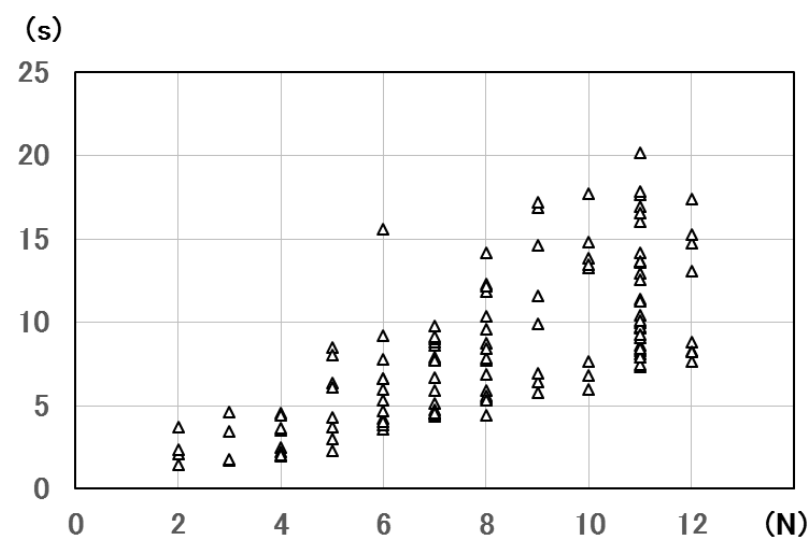

Fig. 8 Process number (N) and communication time (s); Transparent character board

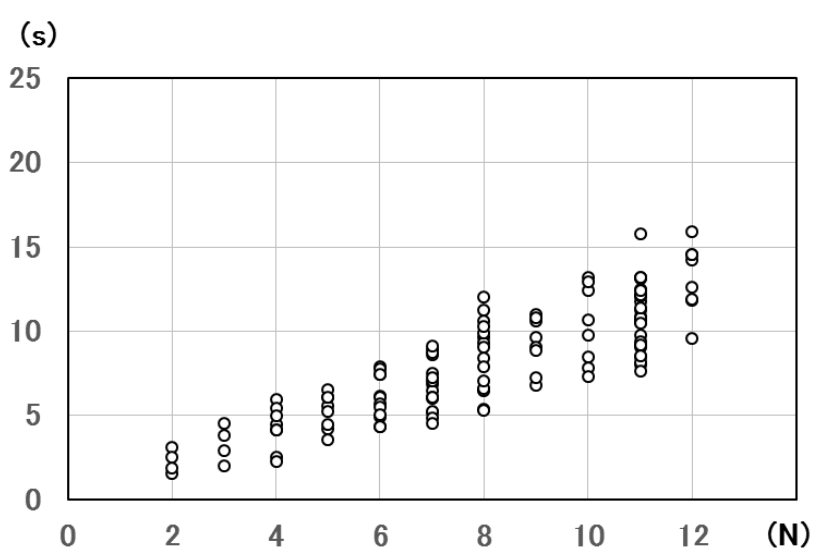

Fig. 9 Process number (N) and communication time (s); Mouth-shape character

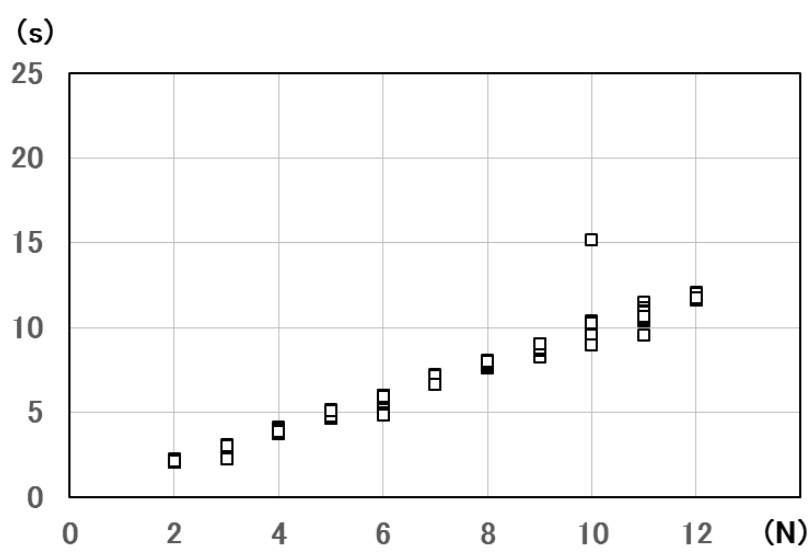

Fig. 10 Process number $(\mathrm{N})$ and communication time (s); Electronic character board

\section{Discussions}

\section{A. Operation process and time}

There was no significant difference in time among the three communication aids according to inferential statistics. This suggests that the increase in time due to the increase in the process number was not large enough to make a significant difference. Therefore, the average values and standard deviations of the time duration for each process number for the 3 communication methods are shown in Table 1 and Fig. 11-13 and are compared.

Table 1 Total communication time for each process number

\begin{tabular}{|c|c|c|c|c|}
\hline $\begin{array}{c}\text { Psocess } \\
\text { (N) }\end{array}$ & $\begin{array}{c}\text { Time } \\
(\mathrm{s})\end{array}$ & $\begin{array}{l}\text { Trasparent } \\
\text { character } \\
\text { board }\end{array}$ & $\begin{array}{l}\text { Mouth- } \\
\text { shape } \\
\text { Character }\end{array}$ & $\begin{array}{l}\text { Electronic } \\
\text { character } \\
\text { board }\end{array}$ \\
\hline \multirow{2}{*}{2} & mean & 2.39 & 2.28 & 2.16 \\
\hline & SD & 0.84 & 0.59 & 0.07 \\
\hline \multirow{2}{*}{3} & mean & 2.88 & 3.33 & 2.82 \\
\hline & $S D$ & 1.21 & 0.95 & 0.34 \\
\hline \multirow{2}{*}{4} & mean & 3.10 & 4.25 & 3.95 \\
\hline & SD & 0.99 & 1.20 & 0.12 \\
\hline \multirow{2}{*}{5} & mean & 5.28 & 5.24 & 4.91 \\
\hline & SD & 2.15 & 0.97 & 0.21 \\
\hline \multirow{2}{*}{6} & mean & 6.44 & 6.06 & 5.70 \\
\hline & SD & 3.20 & 1.24 & 0.38 \\
\hline \multirow{2}{*}{7} & mean & 6.82 & 6.78 & 6.88 \\
\hline & $S D$ & 1.92 & 1.44 & 0.18 \\
\hline \multirow{2}{*}{8} & mean & 8.55 & 8.49 & 7.85 \\
\hline & SD & 2.86 & 2.02 & 0.15 \\
\hline \multirow{2}{*}{9} & mean & 11.17 & 9.26 & 8.84 \\
\hline & $S D$ & 4.35 & 1.48 & 0.24 \\
\hline \multirow{2}{*}{10} & mean & 11.69 & 10.33 & 10.60 \\
\hline & SD & 4.01 & 2.20 & 1.79 \\
\hline \multirow{2}{*}{11} & mean & 11.89 & 10.80 & 10.77 \\
\hline & $S D$ & 3.57 & 1.89 & 0.32 \\
\hline \multirow{2}{*}{12} & mean & 11.69 & 13.15 & 11.85 \\
\hline & SD & 3.63 & 1.89 & 0.13 \\
\hline
\end{tabular}


(s)

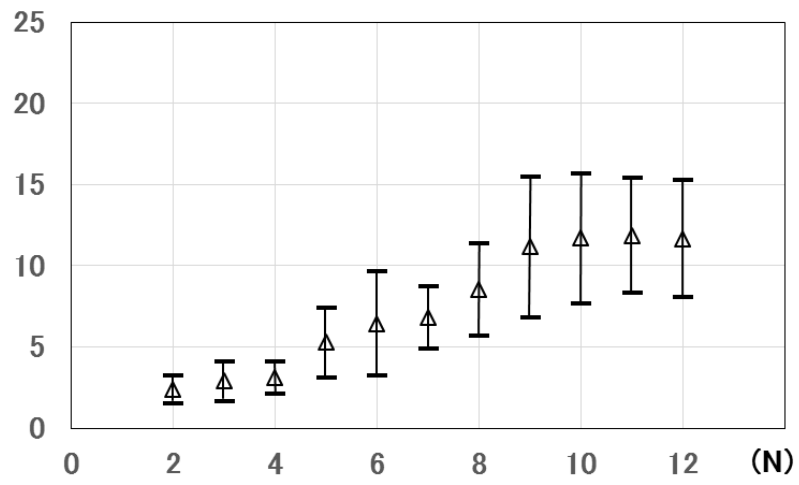

Fig. 11 Total of process number and communication time; Transparent character board

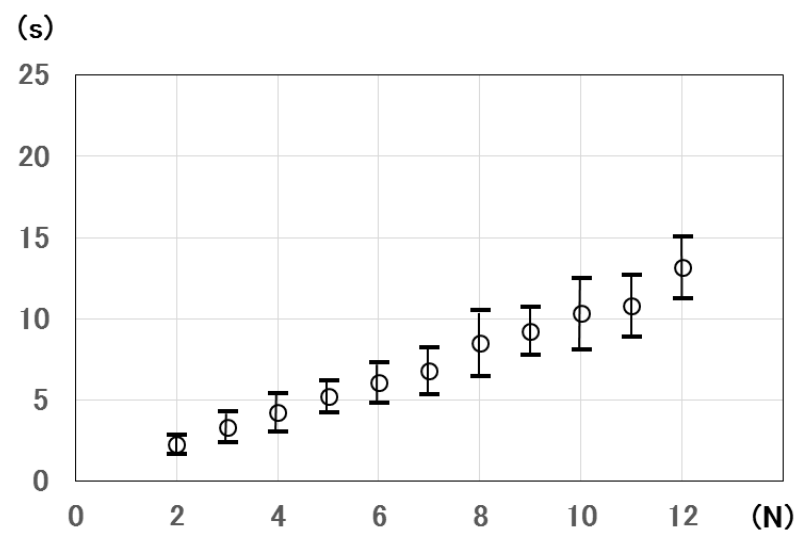

Fig. 12 Total of process number and communication time; Mouth-shape character

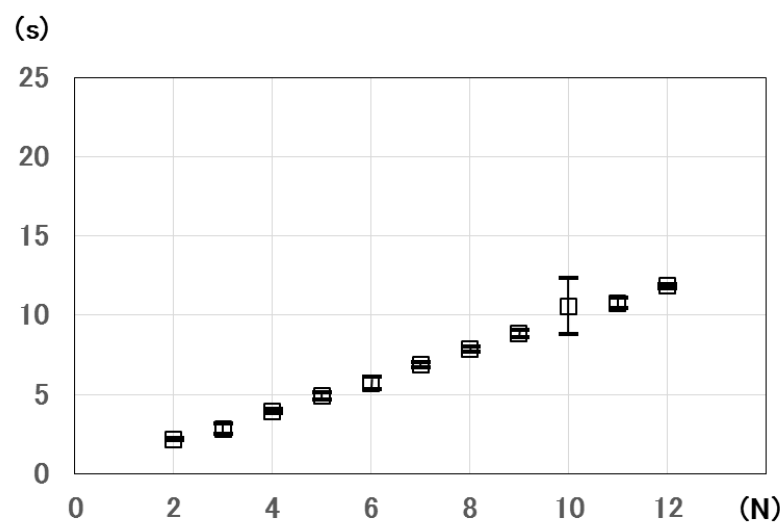

Fig. 13 Total of process number and communication time; Electronic character board

The communication time using the electronic character board as a control (Fig. 13) was about 1s per 1 process. This means that the scan speed was 1 process/s, and it is probable that the patient and the caregiver communicated according to the scan timing from the device. Both the average value and the deviation are small, and judging from the communication time, it can be said that a stable communication was being conducted.

The average communication time using the transparent character board (Fig. 11) was 11s when the process number was 9 to12. In general, the deviation was higher than the other 2 aids. Meanwhile, if we look at the sum of the average value and the minimum of deviation in the time for each process shown in Fig. 10 and the minimum of deviation in the time for each process shown in Fig. 7, there were some trials in which communication could be performed faster than in the other 2 aids.

In the communication time using the mouth-shape character method (Fig. 12), the increase in time due to the increase in the process number was almost linear as in the case of the electronic character board. The deviation increased slightly as the process number increased. The deviation of the mouth-shape character method was larger than that of the electronic character board, but it is considered to be largely due to the utterance of the caregiver in this mouth-shape character method. Furthermore, differences in the caregiver and in the speed and interval of utterances due to repeated trials are suggested.

In order to confirm the degree of deviation in the process of the 3 communication aids excluding the effect of the difference in process number $(\mathrm{N})$, the coefficient of variation $(\mathrm{CV})$ of time in each process of each communication aid was shown (Fig. 14). Since the higher values of the coefficients of variation were found in the transparent character board, it is considered that there was a deviation which originated from this communication method.

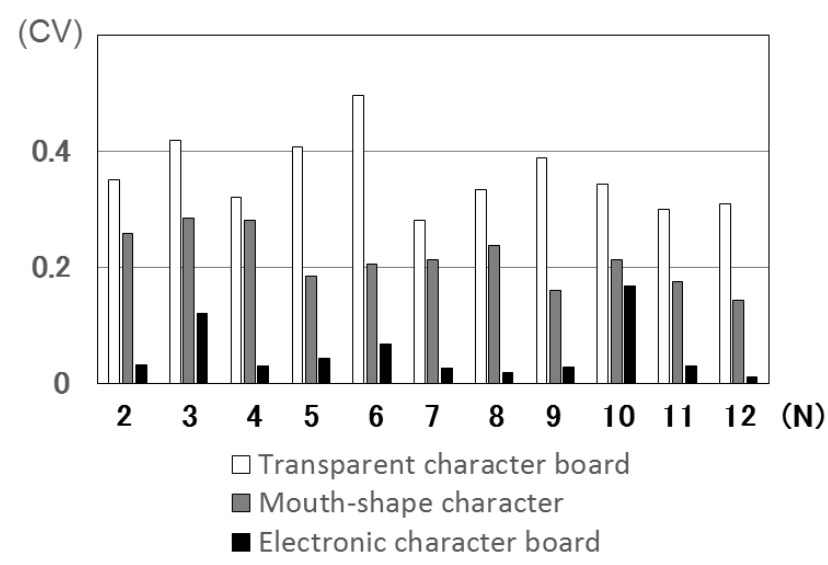

Fig. 14 Coefficient of variation for 3 communication aids

\section{B. Number of failed operational process}

There was no significant difference in the number of failures using the three communication aids according to inferential statistics. However, the number of communication failures using the transparent character board was higher than that of using the mouth-shape character method and of the electronic character board. The cause was examined from the recorded trial video files and the impression of the subjects after the trials. Common causes of all the three aids included failure in responding to the scanning speed, delay in the manifestation of the patient's intention and delay in its confirmation by the caregiver. In addition, in the case of the transparent character board, when the operational process is advanced, the caregiver was not able to determine which of the adjacent character boxes on the board was the target, making the operation of the board ambiguous. 


\section{Caregiver's angle of eye-movement}

The time required and the number of failures in the communication process were examined from the angle of eye-movement of the caregiver. The angle of eye- movement was defined as the combined angle of both eyes [14]. The horizontal and vertical angular ranges of eye-movement in the communication process were determined. One representative case was extracted and compared in each of the three communication aids (Fig. 15-17). The process of "mo" which is one of the character requiring the highest process number $(\mathrm{N}=12)$ was extracted.

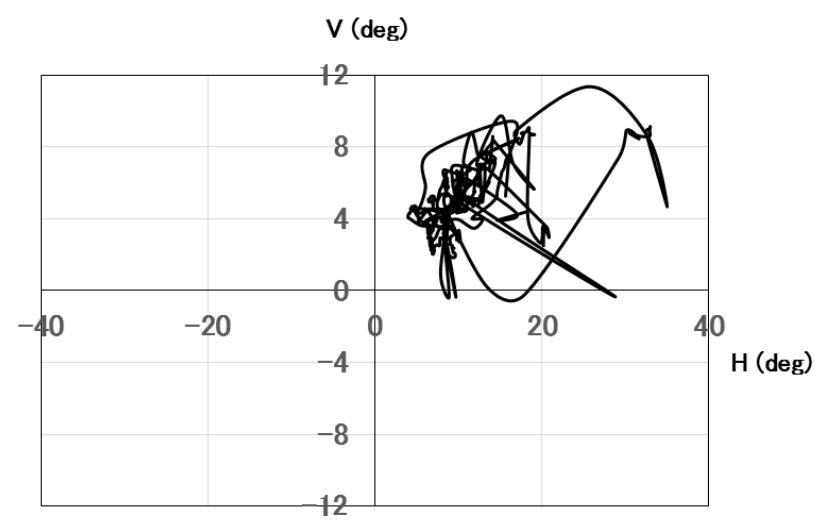

Fig.15 Angle of eye-movement; Transparent character board

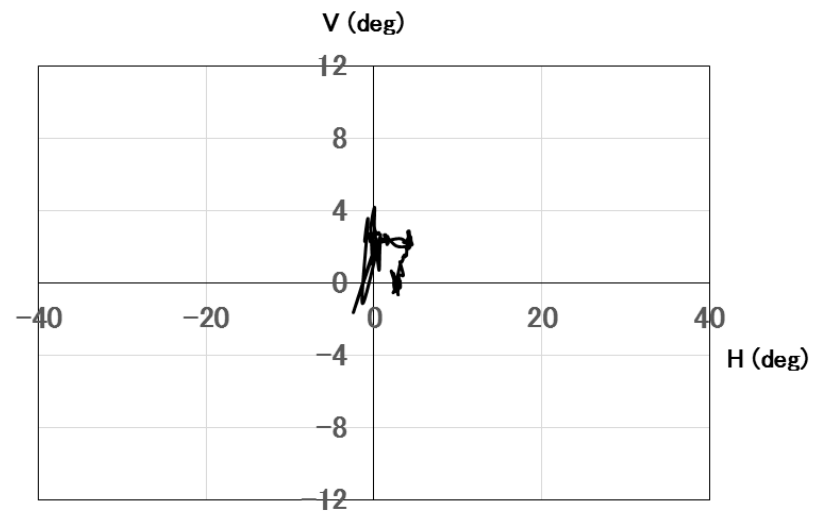

Fig.16 Angle of eye-movement; Mouth-shape character

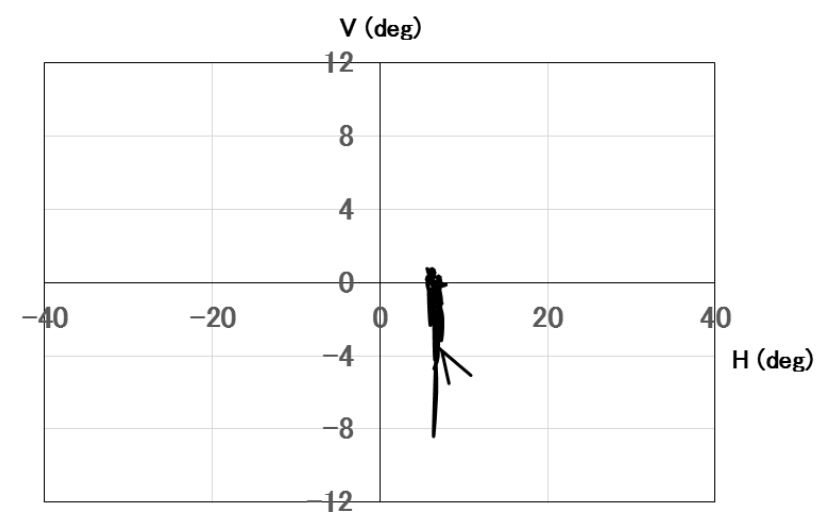

Fig.17 Angle of eye-movement; Electronic character board

In the case of the transparent character board, the horizontal direction was 31.05 degrees and the vertical direction was 11.7 degrees. In the case of the mouth-shape character method, the horizontal direction was 7.02 degrees and the vertical direction was 5.82 degrees. In the case of the electric character board, the horizontal direction was 5.31 degrees and the vertical direction was 9.08 degrees. Of the three aids, the range of angle of eye-movement by the process of the transparent character board was the largest in both the horizontal and vertical directions. When the video recorded from the eye-tracking device (Fig. 18-20) was checked, the

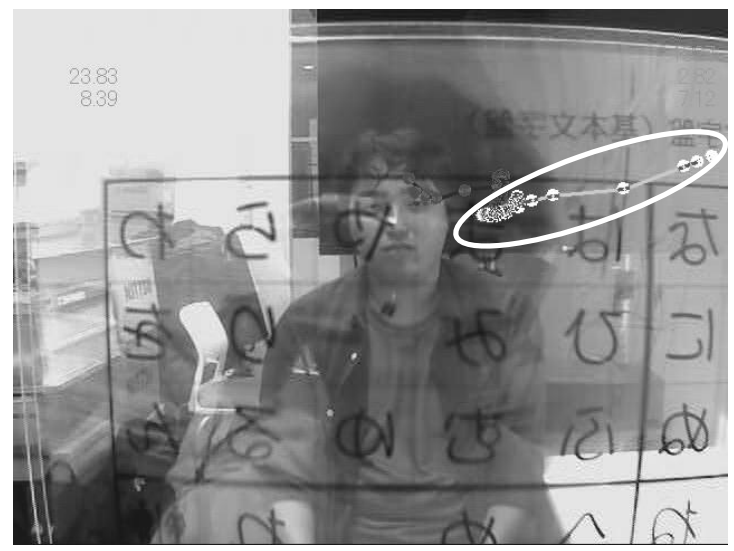

Fig. 18 Eye-movement recording image; Transparent character board

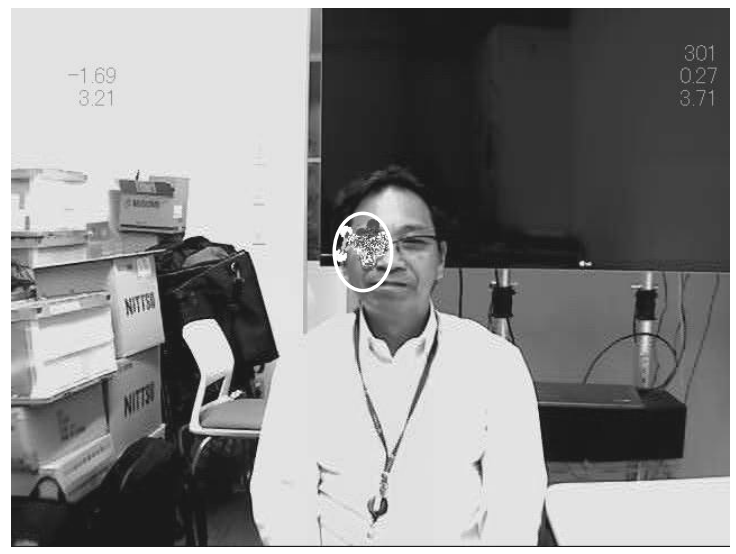

Fig. 19 Eye-movement recording image; Mouth-shape character

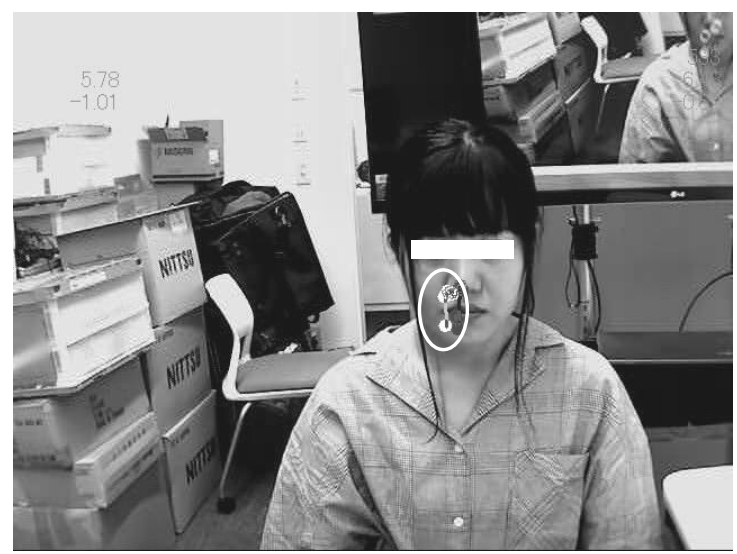

Fig. 20 Eye-movement recording image; Electronic character board

range of eye-movement corresponding to the line of sight range of the caregiver was within the patient's face area in the 
process of the mouth-shape character method and the electronic character board. In the process of the transparent character board however, the range of eye-movement of the caregiver's role was horizontally extended by the movement between the character boxes on the board, and the eye-movement was also extended to the range of the patient face seen beyond the board, thereby confirming the wide range of the movement. Because the communication through the transparent character board was carried out while alternately visually checking the board moving and the facial expression of the patient by the caregiver, it is considered to be difficult to confirm the position of the character boxes. This is suggested to be one of the reasons why this communication aid takes time, is highly variable, and is prone to failure.

\section{Features of the transparent character board and the mouse-shape character method}

Features of the communication aids using the transparent character board and the mouse-shape character method were compared from these experimental results. The transparent character board communication requires the board to be used as a tool. The caregiver and the patient must keep their line of sight consistently for this communication aid, and that the caregiver also needs to alternate between seeing characters on the board and the patient's facial expression. These increase the likelihood of wide eye movement and communication errors. It may be possible, however, to increase the communication speed by further learning operational methods and reducing communication errors. If both patients and caregivers are able to spend time mastering the operational process, it may be used for speedy communication. The mouth-shape character method requires no additional tools for communication. Caregivers and patients may not necessarily need to keep the same line of sight, and the method of communication between them is easy. The caregiver's vocalization speed determines the entire communication speed. The ease of this method makes it applicable even when the relationship between the patient and caregiver is not so long.

\section{E. Limitations of the study}

There are a number of limitations to this study. The subjects were healthy individuals. Hiragana characters employed in the trials did not use all the characters, but random combinations for experiments. The communication was the process of recognizing one character, not creating words or communicating words.

\section{SUMMARY}

The purpose of this study was to evaluate and compare the features of the "transparent character board" and the "mouth-shape character" method, which are convenient communication aids for patients with ALS or other diseases in people who have difficulty in speaking or writing. The transparent character board communication requires the board as a tool. This method can be used for speedy communication if the environment allows the patient and caregiver to spend much time learning the operation process.
The mouth-shape character method requires no tools for communication. The ease of the method makes it applicable even when the relationship between the patient and caregiver is not so long.

In a communication method in which one character is conveyed at a time, it is necessary to record the characters in order to compose a word or a sentence with multiple characters. Caregivers are required to take notes on transparent character board and mouth-shape character, which may take time or cause errors. Therefore, it is considered necessary to take appropriate measures. We will further explore ways to facilitate communication between patients and caregivers in home and nurse care settings.

\section{REFERENCES}

[1] Salameh JS, Brown RH Jr, Berry JD, “Amyotrophic Lateral Sclerosis: Review," Semin Neurol, vol.35(4), 2015, pp.469 - 76

[2] Loeffler JP, Picchiarelli G, Dupuis , Gonzalez De Aguilar JL, "The Role of Skeletal Muscle in Amyotrophic Lateral Sclerosis," Brain Pathol, vol.26(2), 2016, pp.227 - 36.

[3] Neto LL, Constantini AC, Chun RYS, "Communication vulnerable in patients with Amyotrophic Lateral Sclerosis: A systematic review," NeuroRehabilitation, vol.40 (4), 2017, pp.561 - 568.

[4] Mochizuki Y, Hayashi K, Nakayama Y, Shimizu T, Kamide M, Ogino M, Komori T, Hasegawa M, Isozaki E, Nakano I, “ALS patients with ability to communicate after long-term mechanical ventilation have confined degeneration to the motor neuron system," J Neurol Sci, vol.363, 2016, pp.245 - 248 .

[5] Chaudhary U, Xia B, Silvoni S, Cohen LG, Birbaumer N, "Brain-Computer Interface-Based Communication in the Completely Locked-In State,” PLoS Biol, Vol.15(1), 2017, pp.e1002593.

[6] Pandarinath C, Nuyujukian P, Blabe CH, Sorice BL, Saab J, Willett FR, Hochberg LR, Shenoy KV, Henderson JM, "High performance communication by people with paralysis using an intracortical brain-computer interface," Elife. vol.6, 2017, pp.e18554.

[7] Hwang CS, Weng HH, Wang LF, Tsai CH, Chang HT, “An eye-tracking assistive device improves the quality of life for ALS patients and reduces the caregivers' burden," J Mot Behav, vol.46(4), 2014, pp. $233-8$.

[8] Toshiaki Tamura, Mitsuhira Iiguchi, Shinichi Noto, “"Hitorigoto” notes and a special kana characters board improved quality of life in a patient with Shy-Drager syndrome at the end of life," JJSLandHR, vol.12(2), 2015, pp.87 - 93.

[9] Takashi Nakajima, "Assistive technology for supporting communication for patients with incurable and progressive neuromuscular diseases, including transparent character boards, a mouth-shape character method, and an advanced Cybernic Interface device," J. Natl. Inst. Public Health, vol.66 (5), 2017, pp.491 - 496.

[10] Tomoya Miyasaka, Keiko Kawashima, Mamiko Kamoshida, Tadafumi Saga, Toshiaki Tanaka, "Development of Electronic Transparent Communication Boards Using Two Tablets," IJNTR, vol.4(12), 2018, pp. $20-23$.

[11] Tomoe Inomata, Akira Uno, Noriko Haruhara, "Investigation of Cognitive Factors Affecting Reading and Spelling Abilities of Hiragana Characters in Kindergarten Children," The Japan Journal of Logopedics and Phoniatrics, vol.54(2), 2013, pp.122 - 128.

[12] Hirotaka Kobayashi, "Communication Aids for Amyotrophic Lateral Sclerosis," Jpn J Rehabili Med, vol.55(7), 2018, pp.564 - 572.

[13] Nobutomo MATSUNAGA, Hiroshi OKAJIMA, Yuki SHIDA, Daisuke MATSUNO, "Estimation of steering model depending on gazing distance and its evaluation by using driving simulator," Transactions of the JSME (in Japanese), vol.83(851), 2017, pp.1 - 14.

[14] Kazumasa YAMADA, Masatoshi CHIHARA, Daisuke KIMURA, Kiminobu FURUKAWA, Kazuko WATANABE, "Comparison of Eye Movements during Single- and Dual-task Walking," Rigakuryoho Kagaku, vol.33(1), 2018, pp.141 - 144. 